\title{
Towards a Models Traceability and Synchronization Approach of an Enterprise Architecture
}

\author{
Moreira, José Rogério Poggio \\ Department of Computing Science \\ Federal of University of Bahia \\ Salvador, Brasil \\ jose.poggio@ufba.br
}

\author{
Maciel, Rita Suzana Pitangueira \\ Department of Computing Science \\ Federal of University of Bahia \\ Salvador, Brasil \\ ritasuzana@dcc.ufba.br
}

\begin{abstract}
In the context of Enterprise Architecture (EA) modeling, the lack of alignment between the computational models constitutes an organizational problem. The root cause of this problem is the low traceability capacity and the lack of synchronization between the computational models present in the EA. Among the negative impacts related to this problem are, for example, the obsolescence of the models and the difficulty of carrying out analyzes of impacts and decision making in scenarios of organizational changes. Thus, in order to provide the alignment, understanding and adaptation of the corporate environment, from the institutional strategic level to the operational level of Information Technology (TI), focusing on the information systems, an approach is proposed to enable the traceability and synchronization between the computational models. The proposed approach in this paper consists of a: (i) meta-model set comprising the strategic, tactical and operational levels of the EA; (ii) traceability model that supports configuration and change management, through the use of COBIT and ITIL best practices; (iii) transformation process of models that, through the application of the Model Driven Development (MDD), aims to provide the synchronization between the elements of the different models present in an EA.
\end{abstract}

Keywords- Enterprise Architecture; Model Synchronization; Traceability of Models; Alignment between Business and IT; Alignment between Strategy and Business Processes; Strategic Alignment; Business Model.

\section{INTRODUCTION}

An enterprise architecture is the organizing logic of strategy, business processes and IT infrastructure, including information systems, which reflects the integration and standardization requirements of an institution's strategicoperational model, such as the Zachman and TOGAF models that have driven and consolidated, respectively, the works in this area [10][19][22]. An enterprise architecture is formed by levels that are related: (i) strategic; (ii) business processes (tactical); (iii) services and; (iv) IT infrastructure (operational) [19]. In the modeling of enterprise architecture, the relationship between strategy, business processes and information systems can be represented by the alignment of the computational models elaborated within the organization [5]. The models represent instruments to reach and graphically represent the different levels of abstraction of enterprise architecture and to contribute to the achievement and maintenance of the strategic alignment between business and IT, in addition to their unions providing an integrated visualization of an organization's EA [19]. These models, then, represent the achievement of: (i) corporate and IT strategic planning; (ii) the business tactic to achieve the strategic objectives defined in the strategic planning and; (iii) the IT operation, focusing on information systems. However, the isolated construction of models such as the construction of a requirements model, without having a process model and a strategic model previously defined, can result in the elaboration of semantically fragile and non-aligned models with the business. Another relevant point is that if there is no defined strategy and the business processes are not established or are unstructured and without maturity, there is no reason for an institution to start developing its information systems.

In this scenario, to allow a better understanding of this research, this work defines the term alignment as the ability to trace and synchronize the strategic, tactical and operational structures present in the computational models of an organization.

The lack of traceability or its delivery in an inadequate way constitutes a problem and makes it difficult to see and understand how a set of models and their structures are related, which contributes negatively to the analysis of impacts in scenarios of organizational changes [2].

The absence of synchronization between different models (such as business process model and system requirements model) is another problem and results in the obsolescence of these models, which are now outdated and inconsistent, since the change in some element of the model, does not guarantee the updating of the other associated structures. This makes it difficult to maintain business strategy or at least generates a significant impact analysis effort in a scenario of change. In addition, there is still an equally significant probability of occurrence of failures, since the activity of checking the points that can be affected by a possible change, is manual [20].

In this way, it is proposed an approach that has the objective of enabling the synchronization and traceability between the computational models of an EA. The proposed approach consists of: (i) a meta-model set comprising the strategic, tactical and operational levels of the EA; (ii) an 
independent traceability model that supports configuration and change management through the use of COBIT [8] and ITIL [15] best practices and; (iii) the application of Model-Driven Development (MDD), through the construction of a model transformation process, as a way to synchronize elements between different models.

The remainder of this paper is organized as follows: Section 2 presents the related works. Section 3 describes the meta-model set. Section 4 presents de traceability model. Section 5 describes model transformation process. Finally section 6 presents the conclusion of this work.

\section{RELATED WORK}

This work sought to gather relevant contributions, focused on the theme of strategic alignment between business and IT, focusing on the alignment between computational models of enterprise architecture. For this, a bibliographic survey was performed using systematic review techniques. Were considered the 1,500 works that were most relevant to the theme and that were returned by the search. Posteriorly, 96 works were identified from the association of their titles, with the respective theme of the research and the application of filters related to authors relevant to the theme. Of these, only 44 articles were selected, based on the analysis of inclusion and exclusion criteria defined for the research. The study covered the work that was identified during the research and which was published in the last 13 years (2004 to 2016) and was in English and Portuguese. The following databases were searched: (i) Compendex; (ii) Web of Science; (iii) IEEE Xplorer; (iv) Springer e; (v) Elsevier, in addition to the data sources: EJIS, SCIENCEDIRECT, WORLDSCIENTIFIC and AISEL. Also included in the bibliographic collection, from this work, sources of classic information [22] and reference [10]. From the analysis of the works it was possible to answer the following research questions:

- Q1. Are there techniques that align IT and business through traceability and synchronization between computing models at all levels of an enterprise architecture?

A1. There was no identification of studies that performed alignment (traceability and synchronization) between computational models at all levels of EA. However, research was carried out that deals in a theoretical and partial way with the alignment between computational models [1][5][6][7][9].

- Q2. Is there a graphical language pattern to represent the elements present in the models of the different levels of enterprise architecture?

A2. A graphical language pattern has not been identified for the modeling techniques that are used at the levels of an EA, since each level of the EA uses a different modeling technique and even within an EA level, such as the tactical, represented by business processes, exist different languages that can be used to modeling business process (BPMN, EPC, UML) [3][13][16].

- Q3. Are there standard concepts that are used to define the components used in the models of enterprise architecture?
- A3. It was possible to identify a conceptual standard to define the components used in the models of enterprise architecture. At the process level, for example, there are process and activity concepts [13]. At the strategic and operational levels of IT, it was also possible to identify a conceptual standard [1] [5].

The analysis performed in the correlated work identified, showed that information technology is relevant to organizational performance and contributes directly to business operations. However, the lack of alignment between IT and business still prevails. For the analysis of the related works, tree technical criteria were defined. Each criterion represents the alignment between computational models at a certain level of the business architecture. The criterions defined were:

- Criterion I - Alignment between strategic models: that reflects the alignment between strategic corporate and IT maps;

- Criterion II - Alignment between strategic and tactical models: that reflects the alignment between strategic maps and business process models;

- Criterion III - Alignment between tactical and operational IT models: this reflects the alignment between business process models and IT requirements models, necessary for the development of information systems.

In order to improve the analysis of the works, tree attributes were defined for each criterion. Each attribute represents a characteristic that can be treated by an identified criterion. The attributes identified were:

- Theoretical: it should be used when the work theoretically addresses subjects related to the criterion;

- Traceability: should be used when the work deals with traceability between models;

- Synchronization: should be used when the work deals with synchronization between models.

Based on the identified criterion and attributes, the analysis of the studies found that the vast majority of research focuses on the theme related to criterion III. However, the study revealed that even in the theme of concentration (criterion III), the surveys do not cover the alignment in its completeness, because this does not contemplate the attribute of synchronization between models. The topics related to criterion I, as well as criterion II, have been very incipient, containing proposals that discuss their concepts and focus on the extraction of knowledge from strategic and tactical models (strategic maps and business process models) to identify requirements of systems.

The evaluation of the analyzed works indicated that the contributions of [2] and [5] that originated the languages BSC-P and ARMOR can be adapted to allow the closure of the gaps found in the themes associated to criteria I and II, since these works deal theorically traceability between models that are associated with criterion III. The B-SCP, also stands out as a contribution, due to the validation and verification of business requirements models, in terms of business process and strategy, which may allow an adaptation to the reality of the I criteria and II. [1], together with [7] also presented works 
relevant to the topic of this article, when discussing concepts related to strategic maps, and can be used to support research in the first and second criterion.

The architecture model proposed by [6] also provides a relevant contribution and can be evaluated with a view to adapting to a more strategic business view. Considering the above, the evaluation validated the need to study the strategic alignment theme between business and IT, focusing on the computational models of an enterprise architecture, since it can be verified, the absence of work for some aspects (criterion I and II) and lack of completeness in others (criterion III).

Considering the above, the evaluation validated the need to study the strategic alignment theme between business and IT, focusing on the computational models of an enterprise architecture, since it can be seen, the absence of work for some aspects (criterion I and II) and lack of completeness in others (criterion III).

\section{META-MODEL}

The proposed set of meta-models aims to represent the semantics of EA, proposed by Zachman and TOGAF, and enable the tracking and synchronization of the models present in their different levels of abstraction, keeping the EA always aligned and without obsolescence. For this purpose, metamodels were constructed through the Meta Object Facility (MOF) [17], with the purpose of representing the main concepts, characteristics and relationships present in the strategic, tactical and operational levels of an EA.

Each meta-model is composed of: (i) concepts; (ii) aspects and; (iii) relationships. The concepts represent relevant elements that exist within the levels of the EA and are represented by meta-classes. The aspects represent the important characteristics that are related to the identified concepts and are represented by meta-attributes. Already the relationships represent the semantic associations that exist between the concepts present in the meta-models, being represented by associations. The meta-models were also designed to be independent of the technology used to construct the models (strategic, tactical and operational) that are associated with the respective meta-models. The concepts and aspects present in the set of meta-models were adopted from the perspective of techniques and good practices related to strategic planning, process management and software engineering, which, in turn, are associated with the strategic, tactical and operational levels of EA.

A relevant issue in the conception of this set of metamodels is the integrated and holistic approach to modeling (strategic, tactical and IT operational), viewing them as a single organizational model and reinforcing the institution's strategic thinking development (Objectives, strategies, strategic initiatives, processes and system requirements).

Figure 1 presents the proposal of the meta-model of strategic IT alignment, with a focus on information systems. In it, is possible to observe that there are three types of modeling: (i) strategic; (ii) tactic and; (iii) IT operational. These types of modeling are represented, respectively, in the set of meta- models by the packages: (i) MMEstrategic; (ii) MMTactical and; (iii) MMOperational.

To improve comprehension and understanding of the structures present in the meta-models was performed a description of all concepts and aspects present in the set of meta-models. However, this article presents only the detailing of the strategic meta-model, because it is the least addressed in the related works and most relevant within an institution.

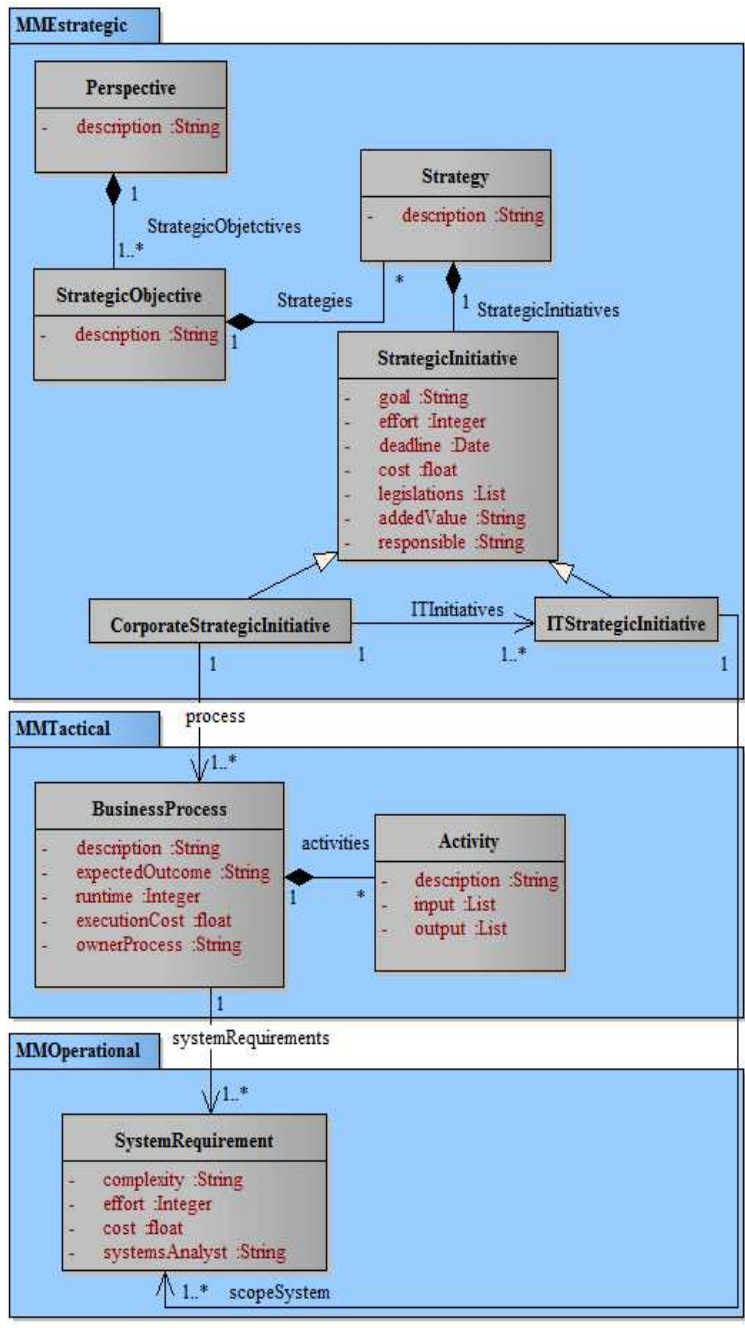

Figure 1. Meta-model Set

- Concept: STRATEGIC INITIATIVE

- Meaning: represents an action, project or program that must be executed to carry out the strategy that has been defined to achieve a strategic objective. A strategic initiative can be corporate or IT.

○ Component of the Meta-Model: meta-class "StrategicInitiative".

- Associate EA level: strategic.

- Good Practice of Origin: BSC and Grumbach [23] [24]. Aspects

- Goal: represents the result that the organization wishes to achieve with the execution of the initiative. This aspect is represented by the metaattribute "objective". 
- Effort: represents the number of hours needed to carry out the initiative. This aspect is represented by the meta-attribute "effort".

- Deadline: represents the date set for the completion of the initiative. This aspect is represented by the meta-attribute "term".

- Cost: represents the financial importance associated with implementing the initiative. This aspect is represented by the meta-attribute "cost".

- Legislation: it represents the law, act or norm directly linked to the initiative and that must be observed during its planning and execution. This aspect is represented by the meta-attribute "legislations".

- Added Value: represents the benefit delivered to the organization by the realization of the initiative. This aspect is represented by the metaattribute "addedValue".

- Responsible: represents the responsible role for the initiative. This aspect is represented by the meta-attribute "responsible".

- Concept: STRATEGY

- Meaning: represents a direction that must be followed by the organization to achieve the defined strategic objective. A strategy can be corporate or IT.

- Component of the Meta-model: meta-class "Strategy".

- Associate EA level: strategic.

- Good Practice of Origin: BSC and Grumbach [23] [24].

- Aspects

- Description: describes in detail the strategy that will be used for the organization to reach the strategic objective (corporate or IT). This aspect is represented by the meta-attribute "description".

- Concept: STRATEGIC OBJECTIVE

- Meaning: represent a result that the organization wants to achieve, being critical for the institution to succeed in its area of operation. A strategic goal can be corporate or IT.

- Component of the Meta-model: meta-class "StrategicObjective".

- Associate EA level: strategic.

- Good Practice of Origin: BSC and Grumbach [23] [24].

- Aspects

- Description: describes the expected result for the strategic objective defined during the strategic planning. This aspect is represented by the metaattribute "description".

- Concept: PERSPECTIVE

○ Meaning: represents the organization's business perspective that, depending on strategic planning (Corporate or IT), is comprised of strategic corporate or IT objectives.
- Component of the Meta-model: meta-class "Perspective".

- Associate EA level: strategic.

- Good Practice of Origin: BSC and Grumbach [23] [24].

- Aspects

- Description: describes the meaning of perspective for strategic planning. This aspect is represented by the meta-attribute "description".

\section{TRACEABILITY MODEL}

This research works with the concept of horizontal and vertical traceability (forward and backward), and may also be associated with low and high granularity. The traceability model proposed in this work is based on several traceability models such as those proposed by [14][18][21].

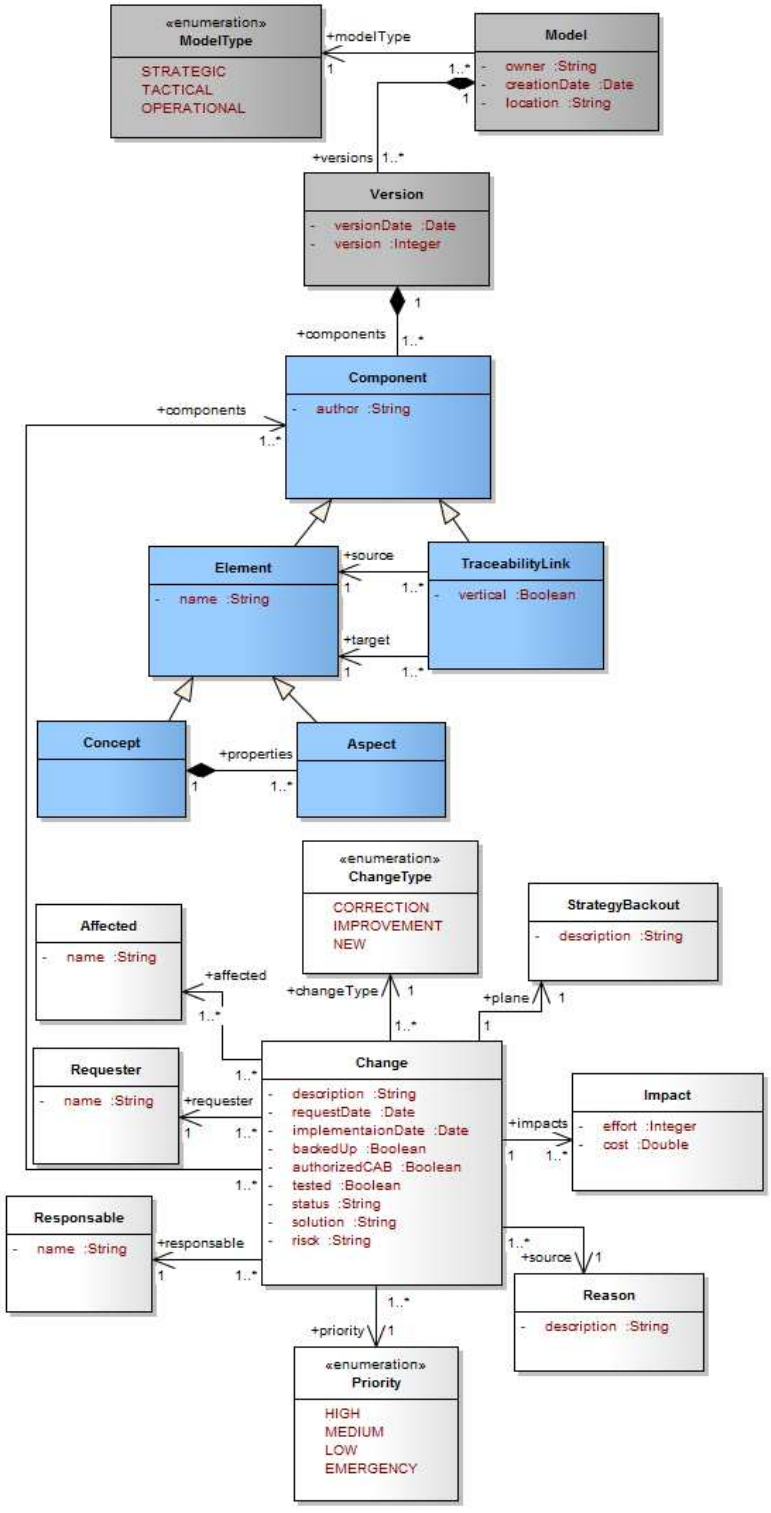

Figure 2. Traceability Model. 
Figure 2 shows the traceability model proposed in this work. The model is divided into three parts. The first part (in blue) represents the structure that allows to trace the elements of the models, present in the different levels of the EA, of vertical or horizontal form, forwards or backwards and in the granularities high and low.

The first part is organized so that a component represents the abstraction of an element or a traceability link between two elements. An element may represent a concept or an aspect, and every traceability link is formed by two elements, one of origin and one of destination. In this way it is possible to create traceability links between concepts, between aspects and between concepts and aspects, besides enabling, as previously mentioned, traceability of low and high granularity, vertical and horizontal.

The second part (in gray) is formed by the configuration management structure. This structure is necessary to identify, control and provide the necessary information about an Organization Configuration Item (CI) [15]. A CI, in the context of this work, is represented by the Class "Model" that represents the strategic, tactical and operational computational models of enterprise architecture, along with its versions, types and components.

The third part (blank) is formed by the change management structure that allows changes to be recorded, prioritized, evaluated and authorized by the Change Advisory Board (CAB), tested and implemented, allowing control over the changes which occur on the models and their components, reducing the risk of incidents and consequently of damages to the organization.

Also according to the elaborated model, a change in a component can generate positive and / or negative impacts for the organization, besides having a requester, a responsible and the affected parties. A change may be requested for the purpose of correcting any problem, for the purpose of functional improvement or inclusion of a new component. In addition, a change always has a reason, which is why the change must be carried out, together with a strategy backout strategy, which is necessary to restore a component to the situation immediately prior to the change.

\section{TRANSFORMATION PROCESS}

In order to synchronize the elements of a set of models, preliminary theoretical studies were performed, demonstrating that the use of the Model-Driven Development (MDD) [12] approach would allow the synchronization of the concepts and aspects present in the models of an EA, allowing, therefore, that the elements of the models are always updated.

With MDD it is possible to transform more abstract models into more specific models. Among these models, there is a set of transformations rules that are applied to achieve the expected result.

The transformations are relevant and many works have been carried out in order to improve them and even automate those [4]. However, most of these works deal only with the transformations between the Platform Independent Model (PIM) and the Platform Specific Model (PSM), leaving behind the transformations that involve the Computation Independent Model (CIM) [12]. For [4], the transformations involving the CIM models are relevant, since it is through this model that an adequate understanding of the business is obtained that will give rise to the requirements that the information system must attend. Therefore, from the business specification represented by the CIM, the requirements of the information systems that make up the PIM must be determined. For this work, the CIM are represented by the strategic and tactical models of an EA. The operational model is a representation of the PIM.

The process aims to carry out transformations of models (strategic, tactical and operational), originated by the set of meta-models proposed in Section 3. The process, according to Figure 3, consists of five activities:

\section{A. Define Transformation Models}

- Description: This process activity aims identify and define the set of models that will be transformed, along with their respective meta-models, that should be used to carry out the transformations project.

- Input: Need to perform model transformation.

- Output: Model along with their respective meta-models.

B. Define Transformation Strategies

- Description: This activity aims to define the transformation strategy that consists of establishing the direction of transformation, which can be unidirectional or bidirectional, along with the type of transformation that may be out-place or in-place.

- Input: (i) model, along with their respective metamodels; (ii) the need to transform of the project of the models.

- Output: Transformation strategy.

\section{Design Transformations}

- Description: This activity aims to perform the specification of model transformations. This activity is responsible for the mapping of the transformations that will be performed, together with the definition of the transformation language that will be used to transform the models and the establishment of orchestration techniques of the model transformations that will be performed.

- Input: (i) model, along with their respective metamodels; (ii) the need to transform project models; (iii) transformation strategy.

- Output: (i) defined transformation language; (ii) transformation mapping; (iii) defined orchestration techniques.

\section{Implement Transformations}

- Description: This activity is responsible for choosing the most appropriate tool and implementation of transformations.

- Input: (i) model, along with their respective metamodels; (ii) the need to transform project models; (iii) transformation strategy; (iv) defined transformation 
language; (v) transformation mapping performed; (vi) defined orchestration techniques.

- Output: defined tool and implemented transformations solution.

\section{E. Execute Transformations}

- Description: This activity refers to the execution, in practice, of the transformation of the models.

- Input: defined tool and implemented transformation solution.

- Output: transformed and synchronized models.

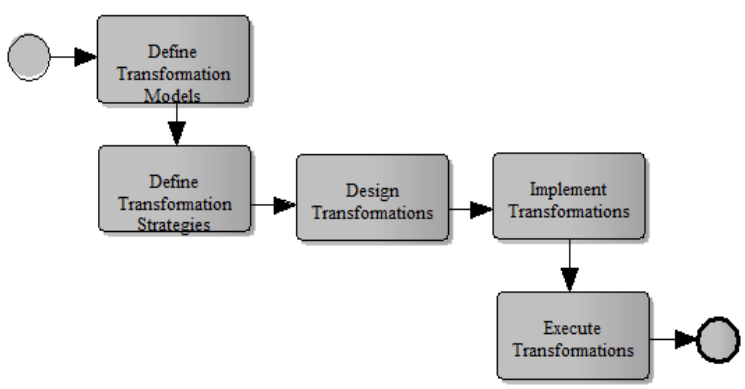

Figure 3. Model Transformation Process.

\section{CONCLUSIONS}

This article presents the proposal that aims to enable the synchronization and traceability of the computational models present in enterprise architecture. The development of this work was carried out based on bibliographic research on the subject of enterprise architecture and under from the perspective of computational models and the strategic alignment of business and IT. At this stage of the work, no studies were found to perform the alignment (traceability and synchronization) of models in their completeness. The possible benefits expected for this research are:

- Creation of the integrated modeling (strategic, tactical and IT operational) approach in a traceable and synchronized way, viewing the models as a single organizational asset.

- Decreased obsolescence of organizational models and, consequently, of the organizational holistic view;

- Improve impact analysis in scenarios of organizational changes, allowing the institution to be more flexible;

- Facilitate the construction and maintenance of information systems.

- Contribute to the achievement of strategic alignment of IT, through by aligning the models present in A.E.

In this way, this work intends not only to improve the way organizations think about performing their computational modeling at the levels of enterprise architecture, but also to improve the way the current tools work with their models.

\section{REFERENCES}

[1]Babar, Abdul., Wong, B., Gill, A. Q., (2011), An Evaluation of the Goal-Oriented Approaches for Modelling Strategic Alignment Concept. Research Challenges in Information Science (RCIS), 1-8. Ieee.

[2]Bleistein, S. J., Cox, K., Verner, J., \& Phalp, K. T., (2006), B-SCP: A requirements analysis framework for validating strategic alignment of organizational IT based on strategy, context, and process. In: Information and Software Technology.

[3]De Sousa, Rafael T.; et al, (2014), Business process modelling: A study case within the Brazilian Ministry of Planning, Budgeting and Management. In: CISTI.

[4]Dias, Felipe.; Morgado, Gisele.; Oscar, Pedro.; Silveira, Denis.; Alencar , Antonio Juarez.; Lima, Priscila. Schmitz, Eber. (2006). Uma Abordagem para a Transformação Automática do Modelo de Negócio em Modelo de Requisitos.

[5]Engelsman, W., Quartel, D., Jonkers, H., \& Sinderen, M. V, 2011, Extending enterprise architecture modelling with business goals and requirements. Enterprise Information System.

[6]Fritscher, B., Pigneur, Y., (2011), Business IT Alignment from Business Model to Enterprise Architecture. Workshop on Business/IT Alignment.

[7]Giannoulis, C., Petit, M., Zdravkovic, J., (2011), Modeling Business Strategy: A metamodel of Strategy Maps and Balanced Scorecards. Research Challenges in Information Science.

[8]ITGI. (2012) COBIT $5 . \quad$ In: <http://www.isaca.org/cobit/Documents/COBIT-5-Introduction.pdf>. Accessed in June 2016.

[9]Jonkers, H., Lankhorst, M., Buuren, R. V., 2004, Concepts for modeling enterprise architectures. International Journal of Cooperative Information Systems.

[10]Josey, Andrew et al. (2011). TOGAF Version 9.1.

[11]Letelier, P. (2002). A framework for requirements traceability in UML-based projects. In International Workshop on Traceability In Emerging Forms of Software Engineering.

[12]Lucrédio, Daniel. (2009). Uma Abordagem Orientada a Modelos para Reutilização de Software. USP - São Carlos.

[13]Kurz, Matthias., (2016)., BPMN Model Interchange: The Quest for Interoperability., 8th S-BPM.

[14]Oglio, Pablo Dall. Silva, João Pablo Silva da. Crespo, Sergio. (2010). Um Modelo de Rastreabilidade com suporte ao Gerenciamento de Mudanças e Análise de Impacto.

[15]OGC. The Official Introduction to the ITIL Service Lifecycle. UK: TSO, 2007.

[16]OMG., 2015, UML 2.5 Specification. In: http://www.omg.org/spec/UML/2.5/PDF/.

[17]OMG., (2016), MOF 2.5.1 Specification. In: http://www.omg.org/spec/MOF/2.5.1/PDF. Accessed in July of the 2016.

[18]Ramesh, B., \& Jarke, M. (2001). Toward reference models for requirements traceability. IEEE Transactions on Software Engineering.

[19]Ross, J. W., Weill, P., Robertson D. C., (2008), Arquitetura de TI como Estratégia Empresarial.

[20]Salgado, Carlos., Machado, Ricardo., Suzana, Rita., (2013), Using Process-level Use Case Diagrams to Infer the Business Motivation Model with a RUP-based Approach.

[21]Sayão, Miriam. Leite, Julio Cesar. Rastreabilidade de Requisitos. (2006). Revista de Informática Teórica e Aplicada.

[22]Zachman, J. a. (1987). A framework for information systems architecture. IBM Systems Journal, 26(3), 276-292.

[23]Brainstorming. Contruindo o Futuro - O Método Grumbach de Gestão Estratégica. 2012. In: <https://www.stm.jus.br/midias-planejamentoestrategico/artigos>. Accessed in June of the 2016.

[24]Kaplan, R. S. e Norton, D. P. - A Estratégia em Ação - Balanced Scorecard. Rio de Janeiro: Campus, 1997. 